\title{
Anesthetic Implications of Robotically Assisted Surgery with the Da Vinci Xi Surgical Robot
}

\author{
John L. Raytis ${ }^{1 *}$, Bertram E. Yuh ${ }^{2}$, Clayton S. Lau' ${ }^{2}$, Yuman Fong' ${ }^{2}$, Michael W. Lew ${ }^{1}$ \\ ${ }^{1}$ Department of Anesthesiology, City of Hope, Duarte, USA \\ ${ }^{2}$ Department of Surgery, City of Hope, Duarte, USA \\ Email: *jraytis@coh.org
}

Received 9 June 2016; accepted 22 August 2016; published 25 August 2016

Copyright (C) 2016 by authors and Scientific Research Publishing Inc.

This work is licensed under the Creative Commons Attribution International License (CC BY).

http://creativecommons.org/licenses/by/4.0/

c) (†) Open Access

\begin{abstract}
Surgeries performed with traditionally available robotic systems have many well-documented anesthetic implications. In this observational report, new and unique anesthetic considerations encountered with the introduction of the da Vinci Xi robot related to positioning operating room equipment, patient access and chance for unintended patient contact are described.
\end{abstract}

\section{Keywords}

Anesthetic Implications, Robotic Surgery, Da Vinci Xi

\section{Introduction}

The annual number of robotic-assisted surgeries performed worldwide has increased from approximately 1000 in the year 2000 to over 450,000 in 2013 [1]. Currently, a wide variety of surgical procedures-urologic, gynecologic, hepatobiliary, colorectal, otolaryngologic, thoracic and cardiothoracic - are performed robotically.

As has been described [2]-[4], robotic-assisted surgery has many implications for the anesthesiologist. Patient hemodynamics and ventilation are affected by the pneumoperitoneum and degree of trendelenburg (or reverse trendelenburg) position needed for the use of a robotic surgical system. Also, docking of the robot may require that the head of the patient be rotated away from the proximity to the anesthesiologist, which affects the position of equipment in the operating room, may reduce airway access and affect the use of arterial, central and intravenous lines. In addition, there may be a need to turn the orientation of the operating room table when converting from a robotic to an open procedure in order to use of fixed lighting designed for open surgery or to admi-

${ }^{*}$ Corresponding author.

How to cite this paper: Raytis, J.L., Yuh, B.E., Lau, C.S., Fong, Y. and Lew, M.W. (2016) Anesthetic Implications of Robotically Assisted Surgery with the da Vinci Xi Surgical Robot. Open Journal of Anesthesiology, 6, 115-118.

http://dx.doi.org/10.4236/ojanes.2016.68019 
nister emergency therapy such as electronic pacing or defibrillation.

The FDA approved the Da Vinci Xi robotic surgical system manufactured by Intutive Surgical, Inc. in April 2014. Prior to acquiring the da Vinci Xi system, the da Vinci S and Si robotic surgery systems were used at our institution since 2003 to perform over 9000 robotic-assisted procedures in urology, gynecology, hepatobiliary surgery, general surgery, otolaryngology and thoracic surgery. Since October 2014, the da Vinci Xi surgical system has been used in over 250 procedures at our hospital. Several important and unique issues relating to anesthetic care have been observed with the use of the da Vinci Xi versus the da Vinci S and Si surgical robots. The purpose of this observational report is to describe new and unique anesthetic implications encountered with the introduction of the da Vinci Xi robotic surgical system at our institution.

\section{Anesthetic Implications}

\subsection{Robot and Operating Room Table Position}

Robotic systems are made up of multiple components-the patient-side unit containing the robotic arms, the imaging tower and the surgeon's operating console. With the da Vinci S and Si surgical systems, the patientside unit is typically brought in at a 45 degree angle relative to the initial position of the operating room table. To accommodate this position of the robot, the operating room table and the patient's head are routinely turned away from their initial position near the anesthesiologist (Figure 1).

By contrast, the patient-side unit of the da Vinci Xi robot is brought in perpendicular to the standard patient position, and the operating room table is less frequently turned relative to the anesthesiologist (Figure 2). This is due to the fact that the robotic arms on the patient-side unit of the da Vinci Xi robot are able to rotate and assume different orientations relative to the base of the unit. The result is that in the head of the bed is near the anesthesiologist for the majority of surgical procedures. The exception to this at our institution is the robotically assisted nephrectomy for which the configuration of operating room equipment is as listed in Figure 1 for both the Xi and the Si and S systems.

Not turning the patient relative to the anesthesiologist reduces the need for extensions to intravenous, arterial and central line tubing and the need to move the anesthesia machine to accommodate connection to the anesthesia circuit and monitors. It also may reduce the chance for displacement or disconnection of lines, the anesthesia circuit and the endotracheal tube. In addition, the setup time needed to disconnect the anesthesia circuit and monitor cables, to unlock and to turn the operating room table and to reconnect equipment is eliminated at both

(a)

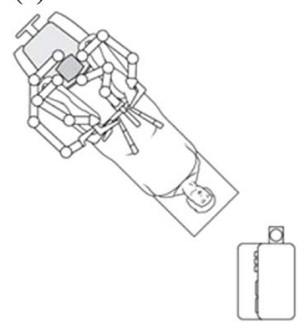

(c)

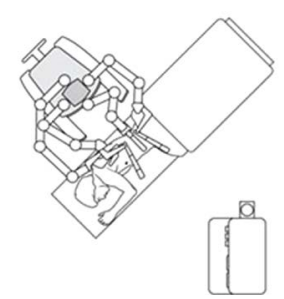

(b)

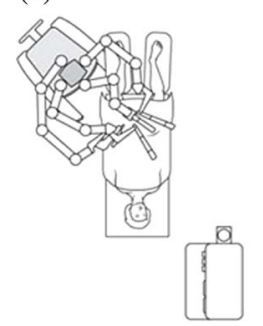

(d)

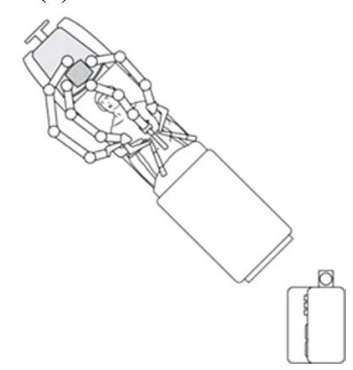

Figure 1. Typical position of operating room equipment for da Vinci S and Si surgical systems. (a) Prostatectomy; (b) Gyenocological surgery; (c) Nephrectomy; (d) Thoracic/hepatobiliary surgery. 


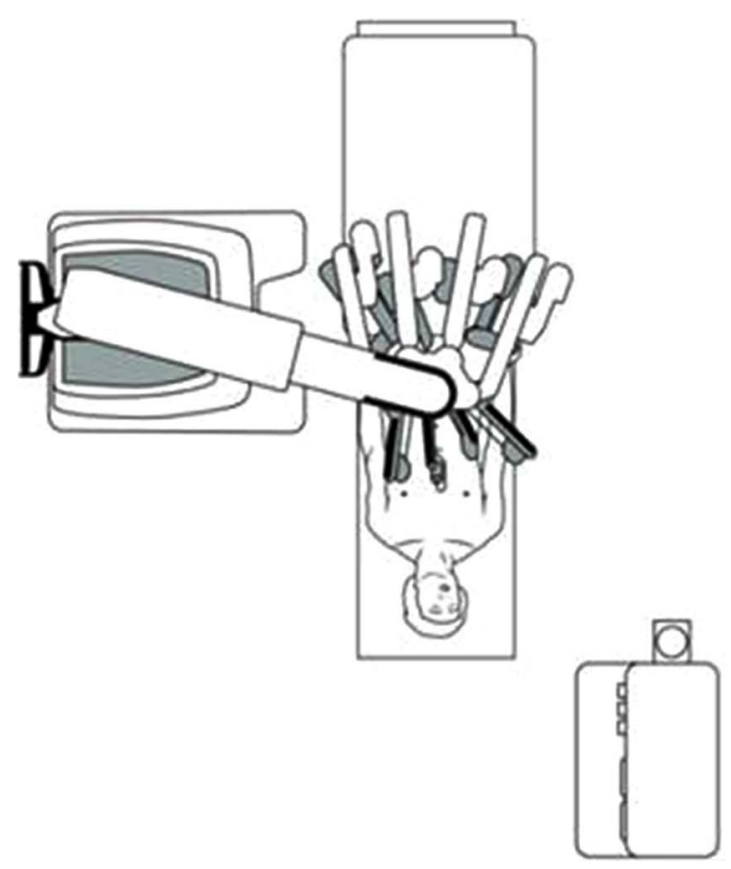

Figure 2. Typical position of operating room equipment for da Vinci Xi surgical system.

the start and end of surgery. Also, there is no need to turn the patient when converting from a laparoscopic to open procedure (in order to utilize fixed lighting designed for open surgery) and when administering emergency therapy such as electronic defibrillation. The ability to convert to an open procedure and to administer emergency therapy quickly without the need to turn the patient may improve patient safety.

\subsection{Patient Access}

Because the majority of surgeries performed with the $\mathrm{Xi}$ robot do not require turning of the operating room table, the anesthesiologist has continuous airway access and ability to monitor the patient's head and eyes routinely during a surgery. Conversely, due of the larger footprint size of the patient-side unit of the Xi robot (1.46 $\left.\mathrm{m}^{2}\right)$ compared to the $\mathrm{S}$ and Si robots $\left(1.15 \mathrm{~m}^{2}\right)$ and due to the physical position of the Xi robot relative to the patient, access to the patient's arm and any line or monitor (intravenous line, arterial line, blood pressure cuff or pulse oximeter) placed on the arm ipsilateral to the patient-side unit of the $\mathrm{Xi}$ robot is reduced relative to such access with da Vinci S and Si systems.

\subsection{Unintended Patient Contact with Robotic Arms}

The robotic arms on the da Vinci Xi robot patient side cart are supported from above as opposed to from the side on the $\mathrm{S}$ and Si robots. Docking with the Xi robot is laser and voice guided and distance from the robotic arm to the patient is greater during surgery on the $\mathrm{Xi}$ robot relative to the $\mathrm{S}$ and Si robots. As a result, the chance for unintended patient contact and trauma appears to be reduced. This is especially true for the third arm on the $S$ and Si robots.

\section{Discussion}

After completing over 250 cases using the new da Vinci Xi surgical system and comparing it to our experience with the da Vinci S and Si systems, several new anesthetic considerations unique to the Xi system have been observed. Because of the larger size of the Xi system, reduced access to the patient's arm ipsilateral to the patient-side unit has been noted. On the other hand, more standardized positioning of operating room equipment for different types of surgeries, the elimination of the need to turn the patient when docking or undocking the patient-side robotic unit and when converting to open surgery or administering emergency therapy, less need for extensions to line tubing and for movement of the anesthesia machine to accommodate connection of the anes- 
thesia circuit and monitors, improved airway access and improved ability to monitor and check the patient's head and eyes, and reduced chance for unintended patient contact and trauma have all been observed with the new Xi system.

\section{Conclusion}

After completing over 250 procedures with the da Vinci Xi surgical system and noting the anesthetic implications, we believe that use of the da Vinci Xi may lead to decreases in operating room times and to increased patient safety. Further studies are needed to validate these initial observations.

\section{Disclosures}

This study was funded institutionally by the City of Hope.

\section{References}

[1] Pinkerton, S. (2013) The Pros and Cons of Robotic Surgery.

[2] Lee, J.R. (2014) Anesthetic Considerations for Robotic Surgery. Korean Journal of Anesthesiology, 66, 3-11. http://dx.doi.org/10.4097/kjae.2014.66.1.3

[3] Berger, J.S., Alshaeri, T., Lukula, D. and Dangerfield, P. (2013) Anesthetic Considerations for Robot-Assisted Gynecological and Urology Surgery. Journal of Anesthesia \& Clinical Research, 345, 517-524.

[4] Hsu, R.L., Kaye, A.D. and Urman, U.D. (2013) Anesthetic Challenges in Robotic-Assisted Urologic Surgery. Reviews in Urology, 15, 178-184.

\section{Submit or recommend next manuscript to SCIRP and we will provide best service for you:}

Accepting pre-submission inquiries through Email, Facebook, LinkedIn, Twitter, etc.

A wide selection of journals (inclusive of 9 subjects, more than 200 journals)

Providing 24-hour high-quality service

User-friendly online submission system

Fair and swift peer-review system

Efficient typesetting and proofreading procedure

Display of the result of downloads and visits, as well as the number of cited articles

Maximum dissemination of your research work

Submit your manuscript at: http://papersubmission.scirp.org/ 